\title{
Die orthopädische Untersuchung des ausgewachsenen Fußes
}

\author{
C. Hase \\ HELIOS Rosmann Klinik Breisach
}

Wie bei allen Körperregionen sind die ausführliche Anamnese und die klinische Untersuchung des Fußes zur Diagnosefindung und zur Einleitung einer weiterführenden Diagnostik unabdingbar. Die zahlreichen anatomischen Strukturen des Fußes sind der manuellen Untersuchung durch den gering ausgeprägten Weichteilmantel gut zugänglich. Dazu sind differenzierte und fundierte anatomische Kenntnisse unbedingt erforderlich sowie die Untersuchungserfahrung, um einen Normbefund von einer pathologischen Auffälligkeit unterscheiden zu können. Obligat ist auch die Untersuchung der nicht betroffenen Gegenseite. Zur zielführenden Diagnostik gehört ein strukturiertes Vorgehen. Dieses Update soll dazu beitragen, eine Untersuchungsabfolge nachvollziehbar durchführen zu können, um zur richtigen Diagnose zu gelangen oder bei unklaren Fällen die Fülle der Möglichkeiten einschränken zu können.

\section{Begriffsbestimmung}

Die bei der Untersuchung des Fußes relevanten Begriffe sind in Tab. 1 definiert.

\section{Anamnese}

Am häufigsten führt die Patienten der Schmerz zur Vorstellung beim Arzt. Allerdings sind auch nicht schmerzhafte angeborene oder erworbene Fehlstellungen mit einer kontinuierlichen oder plötzlichen Veränderung der Fußform Anlass, die Expertise eines Spezialisten einzuholen.

\section{Prinzipien}

\section{Verdachtsdiagnose}

Es ist sinnvoll, die Verdachtsdiagnose dem Patienten jedoch noch nicht mitzuteilen, damit man sich selber alle Möglichkeiten offenhalten kann, den ersten Eindruck durch die folgende Inspektion und körperliche Untersuchung zu erhärten oder zugunsten einer Differenzialdiagnose zu verwerfen.
Oft hat man nach der Anamneseerhebung schon eine Verdachtsdiagnose bzw. es stellt sich die grobe Richtung der Problematik dar.

\section{Abkürzungen}

$\begin{array}{ll}\text { CC } & \text { Calcaneocuboid } \\ \text { D } & \text { Digitus } \\ \text { DIP } & \text { distales Interphalangealgelenk } \\ \text { HV } & \text { Hallux valgus } \\ \text { HZ } & \text { Hammerzehe } \\ \text { IM-Winkel } & \text { Intermetatarsalwinkel zwischen den Metatarsalia } \\ \text { IP } & \text { Interphalangealgelenk } \\ \text { MT } & \text { Metatarsale } \\ \text { MTP } & \text { Metatarsophalangealgelenk } \\ \text { NC } & \text { Naviculocuneiform } \\ \text { OSG } & \text { oberes Sprunggelenk (Tibiotalargelenk) } \\ \text { PIP } & \text { proximale Interphalangealgelenk } \\ \text { TC } & \text { Subtalargelenk (Talokalkaneargelenk) } \\ \text { TMT } & \text { tarsometatarsal } \\ \text { TN } & \text { talonavikular } \\ \text { USG } & \text { unteres Sprunggelenk (TC, TN, CC) }\end{array}$




\section{Grundlagen}

Tabelle 1

Begriffsbestimmung.

\begin{tabular}{|c|c|c|}
\hline Begriff & & Definition \\
\hline Spreizfuß & & $\begin{array}{l}\text { Verbreiterung des Vorfußes durch fächerförmige Aufspreizung des } 1 \text {. und 2. Mittelfuß- } \\
\text { knochens mit Vergrößerung des Intermetatarsalwinkels I zu II von }>12^{\circ}\end{array}$ \\
\hline Hallux valgus & $\mathrm{HV}$ & $\begin{array}{l}\text { Fehlstellung der in sich geraden Großzehe im Großzehengrundgelenk durch lateralisierenden } \\
\text { Zug der Extensor- und Flexorsehnen bei Spreizfuß }\end{array}$ \\
\hline Hallux valgus interphalangeus & & $\begin{array}{l}\text { Fehlstellung der Großzehe im Großzehengrundglied bzw. dem Interphalangealgelenk } \\
\text { mit lateraler Achsenabweichung }\end{array}$ \\
\hline Hallux rigidus & $\mathrm{HR}$ & Einsteifung des Großzehengrundgelenks bei fortgeschrittener Arthrose \\
\hline Hallux limitus & $\mathrm{HL}$ & $\begin{array}{l}\text { Bewegungseinschränkung des Großzehengrundgelenks bei beginnender oder } \\
\text { mittelgradiger Arthrose und/oder Elevation des Metatarsale I }\end{array}$ \\
\hline Hammerzehe & $\mathrm{HZ}$ & flexible oder rigide Flexion im PIP bei Überstreckung im DIP \\
\hline Klauenzehe & & flexible oder rigide Flexion im PIP bei Überstreckung im DIP mit (Sub-)Luxation im MTP \\
\hline Krallenzehe & & $\begin{array}{l}\text { flexible oder rigide Flexion im PIP und DIP mit (Sub)Luxation im MTP, } \\
\text { fehlender Bodenkontakt der Zehenkuppe }\end{array}$ \\
\hline Mallet-Zehe & & flexible oder rigide Flexion im DIP \\
\hline Curly Toe & & nach medial eingerollte, meist noch flexible Zehe im DIP (häufig D IV, V) \\
\hline Schneiderballen & Bunionette & $\begin{array}{l}\text { spiegelbildliches Gegenstück des Hallux valgus mit vergrößertem Intermetatarsalwinkel IV } \\
\text { zu V in Kombination mit einem Digitus quintus varus }\end{array}$ \\
\hline Knick-Senk-Fuß & Pes planovalgus & $\begin{array}{l}\text { Abflachung des Längsgewölbes } \\
\text { Rückfußvalgus } \\
\text { medioplantare Ausrichtung des Talus }\end{array}$ \\
\hline Hohlfuß & Pes cavus/Pes excavatus & $\begin{array}{l}\text { Überhöhung des Längsgewölbes } \\
\text { Rückfußvarus }\end{array}$ \\
\hline Pes metatarsus adductus & & Adduktionsstellung der Metatarsalia (meist MT I, II, III, IV, selten auch MT V) \\
\hline Sichelfuß & Pes adductus & Pes metatarsus adductus mit Rückfußvarus \\
\hline Chopart-Gelenk & & tarsotalare Gelenke (talonavikular und kalkaneokuboid) \\
\hline Lisfranc-Gelenk & & Tarsometatarsalgelenke I-V \\
\hline
\end{tabular}




\begin{tabular}{|l}
\hline Checkliste \\
\hline Anamnese bei Schmerzen \\
\hline Seit wann? (akut/chronisch) \\
\hline Schmerzlokalisation \\
kontinuierlich/intermittierend \\
\hline zunehmend/abnehmend \\
Schmerzcharakter? \\
\hline - stechend \\
- spitz \\
- dumpf \\
- punktuell \\
- flächig \\
- ausstrahlend \\
- kribbelnd \\
- brennend \\
Anlaufschmerz \\
Belastungsschmerz \\
Bewegungsschmerz \\
Ermüdungsschmerz \\
Ruheschmerz \\
Nachtschmerz \\
\hline
\end{tabular}

\section{Inspektion}

Die seitenvergleichende Inspektion des Fußes beginnt mit der Beobachtung des Patienten beim Eintreten. Es schließt sich die Untersuchung unter Belastung am stehenden Patienten, danach im Liegen (Sitzen) an. Dazu sollten immer beide Füße und Beine bis mindestens bis zum Knie sichtbar sein (Hose hochkrempeln). In einigen Fällen ist das Freimachen des gesamten Beins wichtig, d.h. Hose, Strumpfhose, Stützstrümpfe ausziehen, alle Verbände und Pflaster entfernen.

\section{Checkliste}

\begin{tabular}{|l|}
\hline Eigenanamnese des Patienten \\
\hline Fehlstellung angeboren/erworben \\
Trauma einmalig/rezidivierend \\
Trauma direkt/indirekt \\
Unfallhergang akut/länger zurückliegend \\
Vorbehandlung konservativ (welche? wann?) \\
Voroperationen (welche? wann?) \\
Sozialanamnese \\
Beruf \\
Sport (Sportart, Trainingsumfang) \\
Begleiterkrankungen (Diabetes mellitus, Rheuma, \\
Gicht, neurologische Erkrankungen etc.) \\
Medikamente \\
Schuhe/Schuhzurichtungen \\
Einlagen \\
Gehhilfe \\
Bandagen, Schienen \\
\hline
\end{tabular}

Zeitmangel sollte kein Grund sein, auf die Inspektion und Untersuchung beider Füße im Seitenvergleich zu verzichten.

Die Inhalte der Inspektion des Fußes sind in der Infobox „Zielgerichtete Diagnostik“ zusammengefasst.

Wichtig ist auch die Inspektion der Schuhe. Am Trittspurabdruck an Sohle, Innensohle und Einlage sind viele Informationen zu gewinnen (Abb. 3). Wenn Einlagen getragen werden, sollten diese unbedingt mitbeurteilt werden. Dass ein Patient Einlagen trägt, heißt noch nicht, dass es die richtigen sind und sie dem Patienten gut tun. Die Einlagenbeurteilung gehört zur Arbeit des Arztes und sollte nicht nur den Orthopädieschuhmachern überlassen werden. 


\section{Grundlagen}

\section{Zielgerichtete Diagnostik}

\section{Inhalte der Inspektion des Fußes}

- Gangbild

- mit/ohne Schuhe

- flüssig/hinkend

- Fuß- und Beinachse

- im Stehen

- im Liegen

- Zehenstellung

- I. Zehe:

- Hallux valgus

- Hallux varus

- Hallux flexus

- II. Zehe: Zehenlänge (Abb. 1)

- Griechischer Fuß

- Ägyptischer Fuß

- Quadratfuß

- II. - V. Zehe

- Hammerzehen

- Krallenzehen

- Mallet-Zehen

- Curly Toe

- Superduktus-/Infraduktusstellung

- V. Zehe

- Schneiderballen

- Digitus quintus varus

- Vorfußstellung

- Spreizfuß

- breiter/schmaler Fuß

- Abduktion

- Adduktion

- Rückfußstellung

- varus

- valgus

- rectus
- Fußlängsgewölbe

- normal

- abgeflacht

- überhöht

- Fußstellung

- Knick-Senk-Fuß

- Hohlfuß

- Sichelfuß etc.

- „Too-many-Toes-Zeichen“ (Abb. 2)

- Hautveränderungen

- plantar

- Hornhautschwielen

- Druckstellen

- Ulzera (Malum perforans)

- Warzen

- Rheumaknoten

- Morbus Ledderhose

- dorsal

- Clavi (Hühneraugen)

- gesamte Haut

- Rötung, Schwellung, Überwärmung

- Hämatom, Prellmarken

- Narben

- Behaarung

- Pigmentstörungen

- Psoriasis

- Mykose

- Varizen

- Ödeme etc.

- Nagelveränderungen 


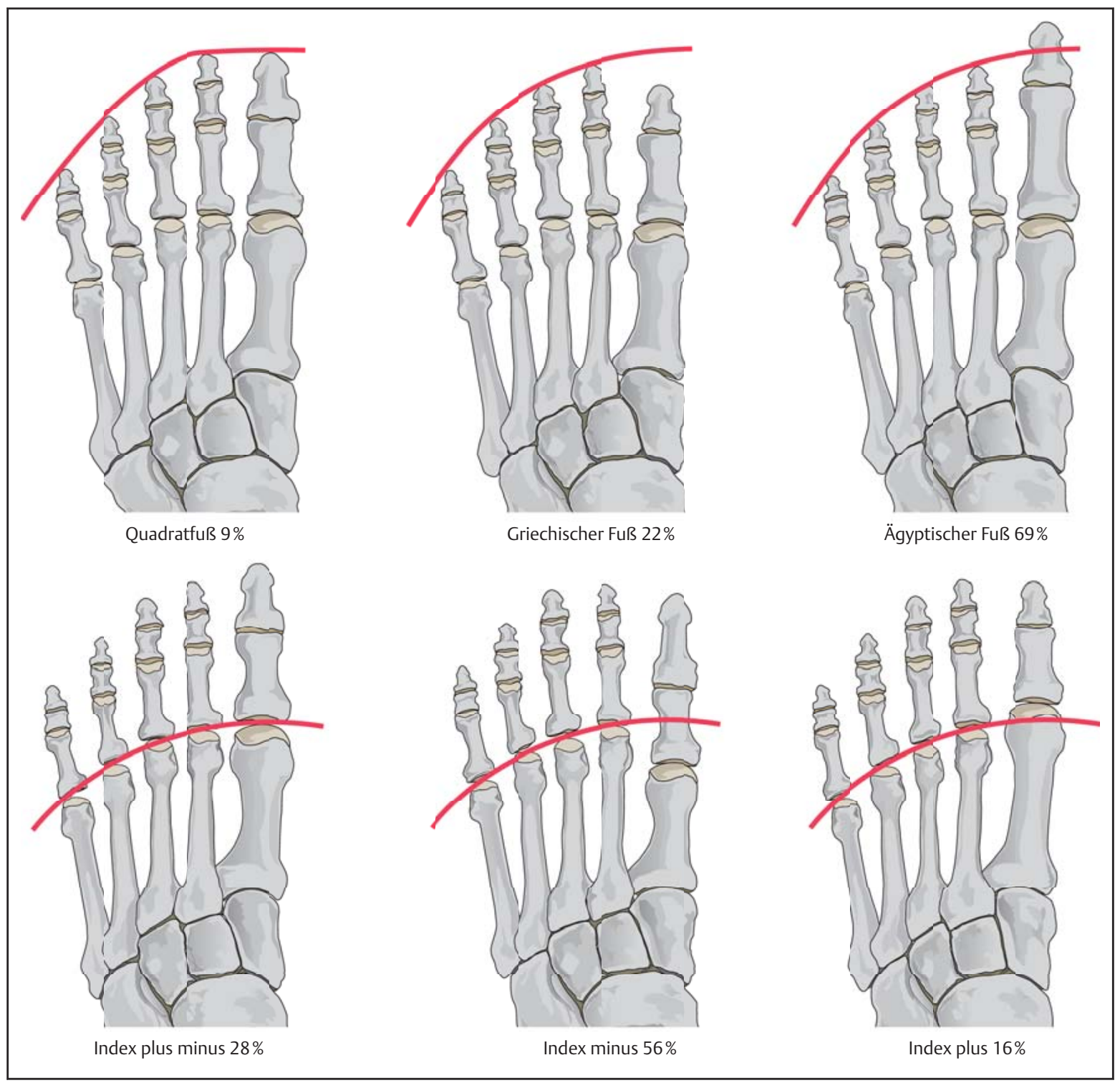

Abb. 1 - Digital- und Metatarsalindex nach Viladot [1].

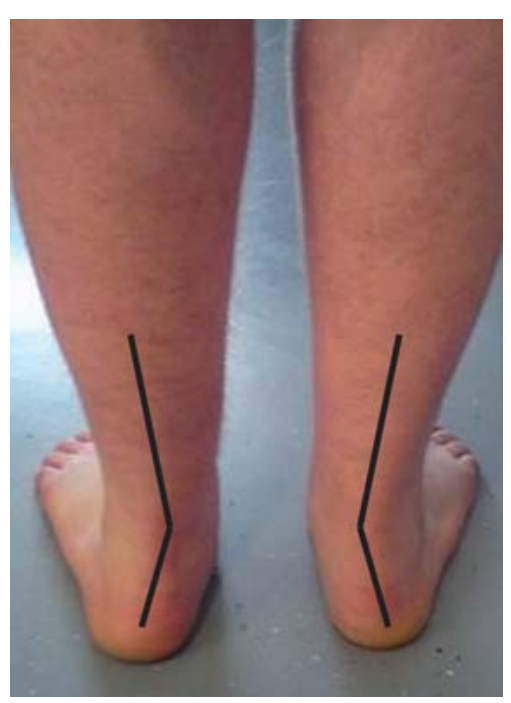

Abb. 2 - „Toomany-Toes-Zeichen". Bei KnickSenk-Fuß mit Rückfußvalgus sind von hinten die lateralen Zehen sichtbar.

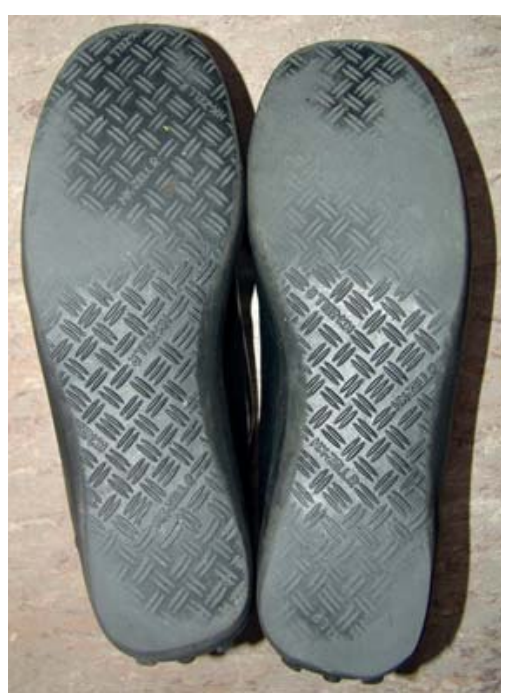

Abb. 3 - Der linke Schuh zeigt sich im Mittelfußbereich gleichmäßig abgelaufen. Am links abgebildeten Schuh ist am MetatarsaleI-Ballen keine Abnutzung zu sehen. Es liegt eine fortgeschrittene Großzehengrundgelenkarthrose vor, bei welcher der Patient keine Last mehr über das MTP I ausübt. 


\section{Grundlagen}

\section{Palpation}

Mit etwas Übung ist es ohne Weiteres möglich, am schlanken Fuß alle Knochen und Gelenke des Fußes bis zum Sprunggelenk zu ertasten. Wichtig ist dabei ein schematischer Untersuchungsablauf, der bei jeder Untersuchung gleich und an beiden Füßen stattfinden sollte.

Es ist einfach, beim liegenden Patienten beide Füße gleichzeitig und synchron zu untersuchen.

Stellen, die der Patient zuvor als besonders schmerzhaft angegeben hat, sollten bis zum Ende der Untersuchung aufgespart werden.

Der Patient wünscht sich zwar, sofort sein Leid zu demonstrieren, aber für uns als Untersucher ist es auch wichtig zu wissen, was nicht schmerzt.

Nach dem Auslösen eines heftigen Schmerzes ist diese Differenzierung in naher Umgebung oft nicht mehr möglich.

Die Inhalte der Palpation des Fußes sind in der Infobox „Zielgerichtete Diagnostik“ zusammengefasst.

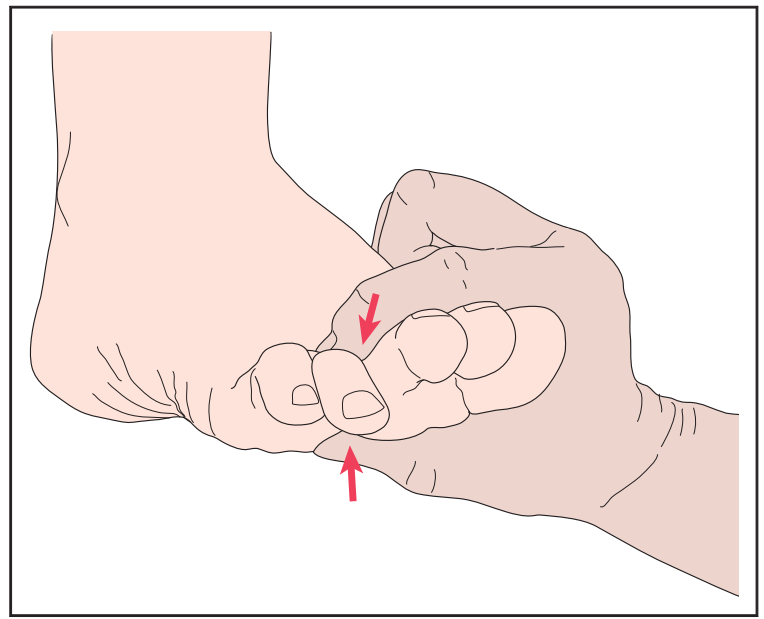

Abb. 4 - Intermetatarsaler Drucktest bei Verdacht auf Morton-Neurom (nach [2]).

\section{Zielgerichtete Diagnostik}

\section{Inhalte der Palpation des Fußes}

- Lokalisation der Schmerzpunkte (vom Patienten zeigen lassen; u. U. Markierung mit Stift)

- Länge und Lage der Zehen und der Mittelfußknochen (Abb. 1)

- Beschaffenheit der Fußsohle - plantares Fettpolster („Fußmatratze“)

- Metatarsalgie bei Druck auf die Metatarsalköpfe

- Palpation der Region zwischen den distalen Metatarsalia

- Morton-Neuralgie zwischen MT II/III und III/IV (Abb. 4)

- Palpation der Gelenke

- Schwellung?

- Erguss?

- Überwärmung?

- Instabilität?

- Pseudoexostose am medialen Metatarsale I

- Bursitis

- akut/chronisch?

- gut tastbare Sehnen- und Faszienansätze und Verläufe:

- Metatarsale-V-Basis: M.-peronaeus-brevis-Sehne, im Verlauf M.-peronaeus-longus-Sehne

- medioplantares TMT I: M.-tibialis-anterior-Sehne

- Tuberositas ossis navicularis: M.-tibialis-posteriorSehne

- Tuber calcanei: Achillessehne

- Processus medialis tuber calcanei: Plantarfaszie

- Sesambeine

- Ansatz der M.-flexor-hallucis-brevis-Sehnen am proximalen Pol des medialen und lateralen Sesambeins

- Sinus Tarsi

- Tarsaltunnel

- A. tibialis posterior

- N. tibialis

- M.-tibialis-posterior-Sehne

- Hauttemperatur

- Hautfeuchtigkeit 


\section{Gefäßstatus}

Untersucht werden

- A. dorsalis pedis,

- A. tibialis posterior,

- A. fibularis (peronaea).

\section{Funktionsuntersuchungen}

Zur Funktionsuntersuchung gehört die Feststellung des Bewegungsumfangs in den Gelenken des Rück- und Vorfußes. Die Funktion der großen Fuß- und Sprunggelenksmuskulatur ist in Tab. 2 dargestellt.

Außer im OSG reicht es, an den anderen Gelenken des Fußes die Angaben in Bruchteilen der normalen Beweglichkeit anzugeben. Trotzdem sind gelegentlich auch Angaben zur Beweglichkeit des USG und des Großzehengrundgelenks zu finden (Abb. 5; Tab. 3).

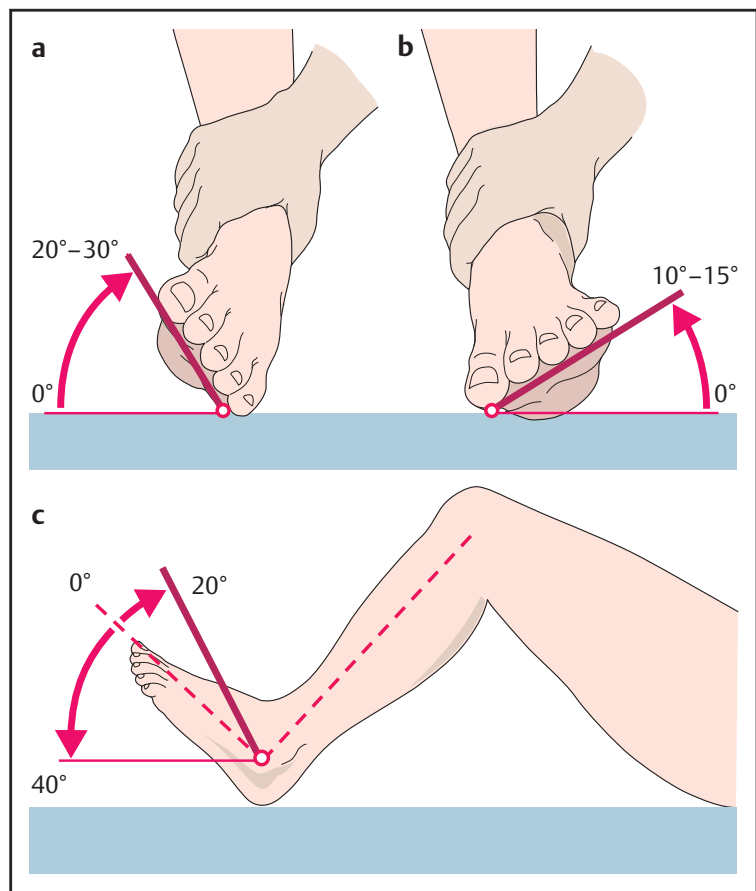

Abb. 5 - Bewegungsumfang im oberen und unteren Sprunggelenk. a Bewegungsumfang als Kombinationsbewegung USG: Inversion/ Eversion (Supination/Pronation). b Bewegungsumfang als Kombinationsbewegung USG: Inversion/Eversion (Supination/Pronation). c Bewegungsumfang OSG: Dorsalextension/Plantarflexion.

\section{Tabelle 2}

Funktion der großen Fuß- und Spunggelenkmuskulatur.

\begin{tabular}{|l|l|}
\hline Funktion & beteiligte Muskeln \\
\hline Dorsalextension & M. tibialis anterior \\
M extensor hallucis longus & M. extensor digitorum longus \\
\hline Plantarflexion & M. triceps surae \\
- M. gastrocnemius pars medialis und lateralis \\
- M. soleus \\
M. peronaeus longus und brevis \\
M. flexor hallucis longus \\
M. flexor hallucis brevis \\
M. tibialis posterior \\
\hline M. peronaeus longus und brevis \\
\hline M. extensor digitorum longus \\
\hline Pronation (Eversion)
\end{tabular}

\section{Tabelle 3}

Aktive und passive Beweglichkeit in den Fuß- und Sprunggelenken.

\begin{tabular}{|c|c|c|}
\hline Gelenk & Beweglichkeit & \\
\hline $\begin{array}{l}\text { oberes Sprunggelenk } \\
\text { (tibiotalar) }\end{array}$ & Dorsalextension/Plantarflexion & $20-0-40^{\circ}$ \\
\hline $\begin{array}{l}\text { untere Sprunggelenke } \\
\text { (talokalkanear, talonavikular, } \\
\text { kalkaneokuboid) }\end{array}$ & $\begin{array}{l}\text { gesamte Beweglichkeit } \\
\text { Eversion/Inversion, Pronation/ } \\
\text { Supination }\end{array}$ & $\begin{array}{l}\text { in Bruchteilen der } \\
\text { normalen Beweglichkeit }\end{array}$ \\
\hline Großzehengrundgelenk & Dorsalextension/Plantarflexion & $70-0-40^{\circ}$ \\
\hline $\begin{array}{l}\text { Großzehenendgelenk } \\
\text { und kleine Zehen }\end{array}$ & & $\begin{array}{l}\text { in Bruchteilen der } \\
\text { normalen Beweglichkeit }\end{array}$ \\
\hline
\end{tabular}




\section{Grundlagen}

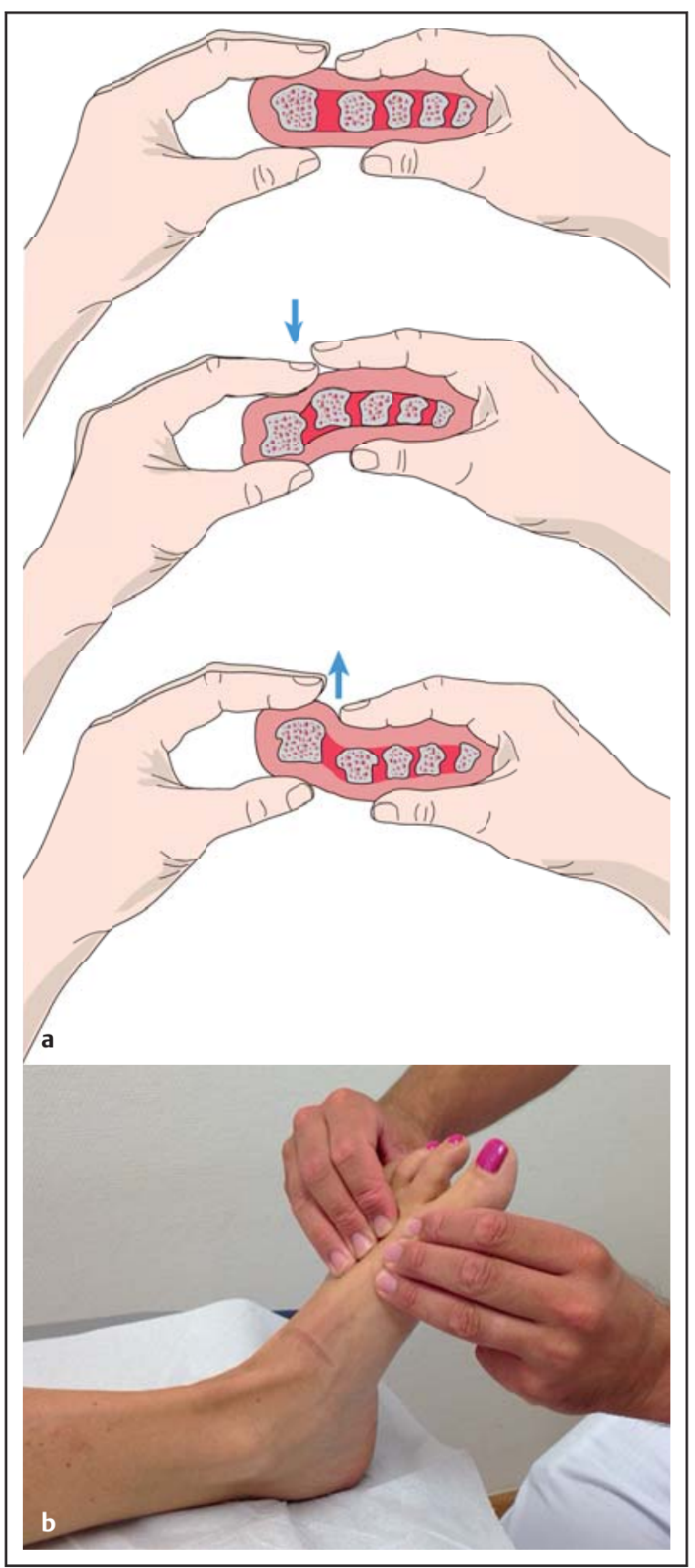

Abb. 6 - Mobilitätsprüfung des I. Strahls in der Sagittalebene. Eine Hand fixiert das Metatarsale II, die andere Hand bewegt das Metatarsale I nach oben und unten. Im Normalfall sollte die dorsale und plantare Mobilität im TMT I gleich sein und $<10 \mathrm{~mm}$ betragen. a Schematische Darstellung (der Pfeil markiert das TMT-I-Gelenk). b Durchführung.

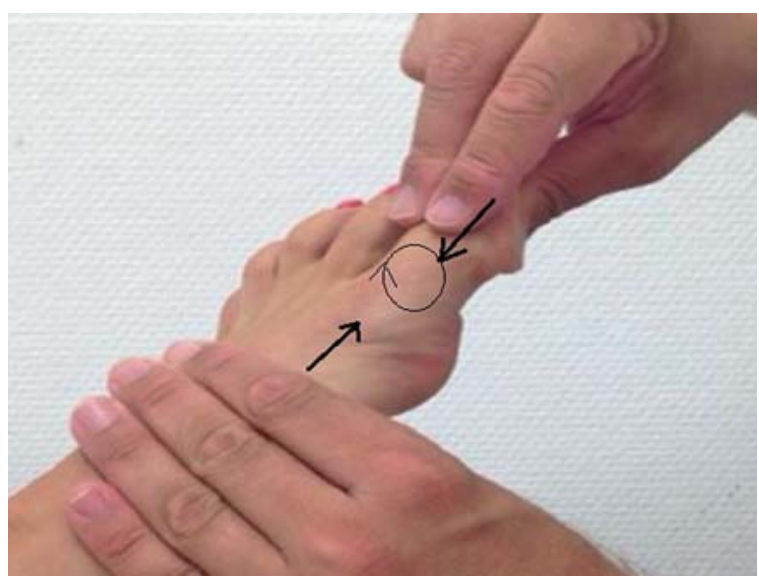

Abb. 7 - Grind-Test: Druck auf das MTP I ausgeübt und Kreisbewegung.

Die Beweglichkeit im Bereich der Fußwurzel - also zwischen Chopart- und Lisfranc-Gelenk - zu beurteilen, ist nicht möglich, da straffe Kapsel-Band-Verbindungen hier ein stabiles Gerüst herstellen und die Gelenke zu rigiden Amphiarthrosen werden lassen. Ausnahme ist die Stabilitätsprüfung des 1. Tarsometatarsalgelenks (TMT). Die Stabilität oder Mobilität dieses Gelenks ist mitursächlich für die Entstehung des Spreizfußes.

Das Ergebnis dieser Untersuchung fließt auch in die Entscheidung für die Wahl eines eventuell notwendigen Operationsverfahrens ein. Richtungsweisend ist hier das Ausmaß der Beweglichkeit in dorsoplantarer Richtung. Hierzu umfasst und stabilisiert der Untersucher das Metatarsale II mit einer Hand, während er das I. Metatarsale in dorsoplantarer Richtung verschiebt (Abb. 6).

Da es kein exaktes Maß für diese Untersuchung gibt und nur die grobe Angabe, dass ein Ausschlag von mehr als $10 \mathrm{~mm}$ pathologisch ist, erfordert die Beurteilung sehr viel Erfahrung. Mit entsprechender Übung kann man auch einen harten Anschlag mit festem Endgefühl bei erhaltenem Kapsel-Band-Apparat von einer weichen Bewegung bei Hyperlaxität oder nach Kapsel-Band-Rupturen unterscheiden.

Bei Vorliegen von arthrotischen Veränderungen können bei der Bewegungsüberprüfung oft Krepitationen ertastet werden. Schon die Extensions- und Flexionsprüfung, aber vor allem der Grind-Test (Abb. 7) geben Aufschluss über den Zustand des Knorpels. Am Großzehengrundgelenk übt man mit der Hand axialen Druck auf das Grundglied aus und führt kreiselnde Bewegungen auf dem Mittelfußknochenkopf durch. Provoziert man hierdurch Schmerzen und Crepitatio, ist von einem Knorpelschaden auszugehen. 


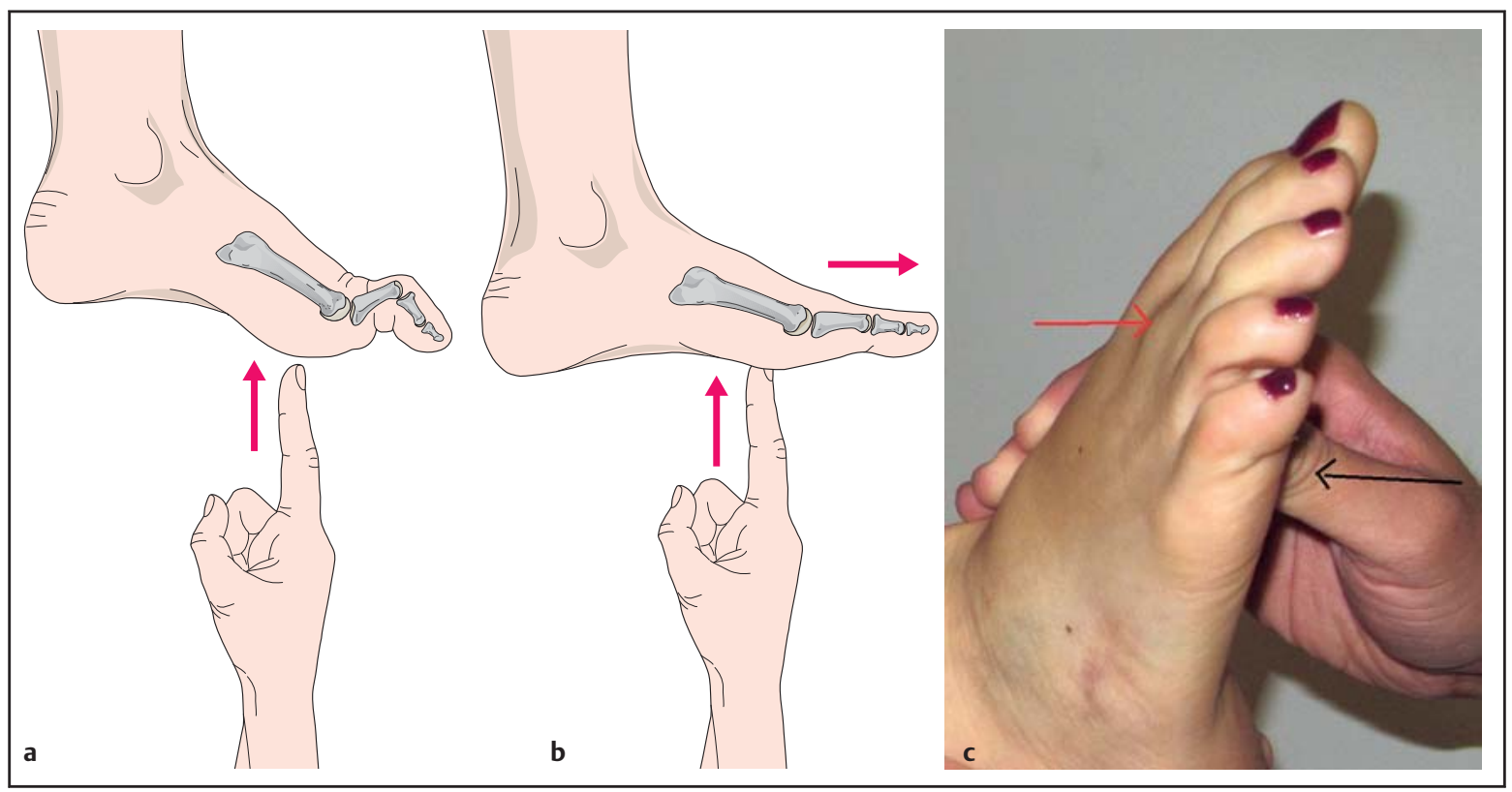

Abb. 8 - Push-up-Test. a Hyperextension im MTP-Gelenk und Hyperflexion im PIP-Gelenk. b Ausgleich der MTP- und PIP-Gelenkfehlstellung durch Druck auf hinter die MT-Köpfe. c Praktische Durchführung.

Der Push-up-Test (Abb. 8) simuliert den Fuß unter Belastung. Drückt man mit einem oder beiden Daumen hinter die mittleren Mittelfußknochenköpfe, sollten sich die Grundgelenke im Normalfall orthrograd ausrichten. Passiert das nicht, muss man von einer (Sub-)Luxation ausgehen. In den meisten Fällen liegt dann eine akute oder schon länger bestehende Ruptur der plantaren Kapselstrukturen vor, die durch die plantare Platte, welche ein Ausläufer der Plantarfaszie ist, verstärkt werden.

Da die Grundgelenke der Kleinzehen nicht - wie an der Hand - deutlich sichtbar sind, sind sie nicht im Bewusstsein der Patienten, sodass Schmerzen in den Kleinzehengrundgelenken oft schwer zuordenbar sind.

Hat man nach dem Push-up-Test den Verdacht einer Instabilität in den Grundgelenken, wird ein dorsoplantarer Schubladentest durchgeführt. Das kann für den Patienten schmerzhaft, aber auch sehr beeindruckend sein, da man die Luxation und die anschließende Reposition im Gelenk gut spürt.

Zur Demonstration für den Patienten und zur eigenen Übung sollte man alle Grundgelenke prüfen, um ein Gefühl für das natürliche Gelenkspiel im Vergleich zu einer Instabilität zu entwickeln.

Manchmal liegt schon lange eine Luxation in einem oder mehreren Grundgelenken vor. Dann sind die betroffenen Zehen unter Umständen nicht mehr reponierbar.
Zur Überprüfung einer Verkürzung der Achillessehne und/oder der Wadenmuskulatur dient der SilfverskjöldTest (Abb. 9). Dabei wird die Dorsalextension im oberen Sprunggelenk sowohl in Kniestreckung (M. gastrocnemius) als auch in Kniebeugung (M. soleus) überprüft. Eine normale Dorsalextension des Fußes im oberen Sprunggelenk bei gebeugtem Kniegelenk und eine Spitzfußstellung bei gestrecktem Knie weisen auf eine Verkürzung des M. gastrocnemius hin. Bei Knick-SenkFüßen ist regelmäßig eine Verkürzung festzustellen.

\section{Fehlstellungen}

Bei Knick-Senk-Füßen ist zudem zwischen rigiden (nicht aktiv oder passiv aufrichtbaren) oder flexiblen (passiv und aktiv oder nur passiv aufrichtbaren) sowie angeborenen oder durch eine Tibialis-posterior-Insuffizienz erworbenen Fehlstellungen zu unterscheiden. Ist der Patient in der Lage, das Längsgewölbe im einseitigen Zehenspitzenstand Single-Heel-Rise-Test (Abb. 10) aufzurichten und der Fersenvalgus gleicht sich aus, ist der Knick-Senk-Fuß flexibel und die M.-tibialis-posteriorSehne intakt.

Fehlt die aktive Kraft zum Aufrichten bei passiver Ausgleichbarkeit im Liegen und treten gleichzeitig Schmerzen im Verlauf der M.-tibialis-posterior-Sehne auf, liegt eine Insuffizienz II. Grades nach Johnson u. Storm vor (Tab. 4; s.a. Tab. 5). Bei fehlender Aufrichtung im Zehenspitzenstand und im Liegen ist der Fuß rigide, dies ist bei 


\section{Grundlagen}

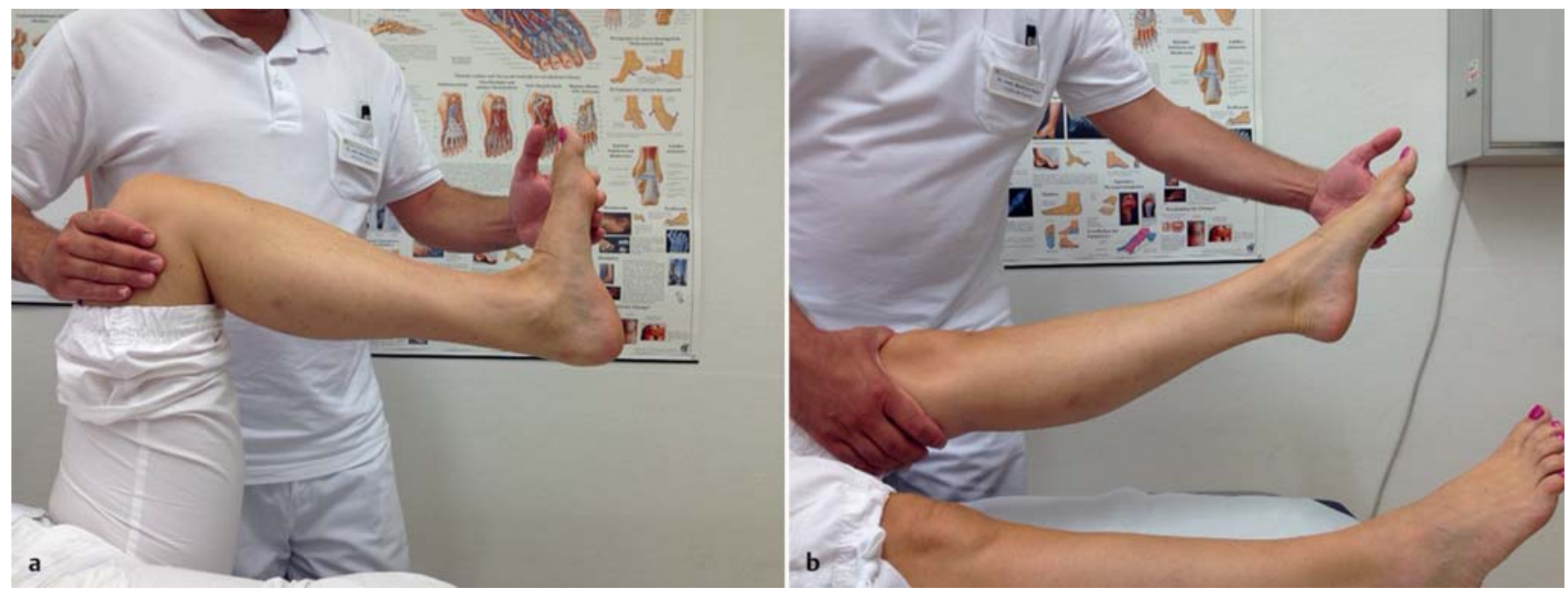

Abb. 9 - Silfverskjöld-Test. Praktische Durchführung: Der Patient wird in Rückenlage mit 90-Flexion im Hüft- und Kniegelenk gelagert. Die Gegenseite ist gestreckt. a Zuerst Prüfung der Dorsalflexion im OSG bei gebeugtem Knie. b Dann Kniestreckung und nochmalige Messung der Dorsalflexion im OSG.
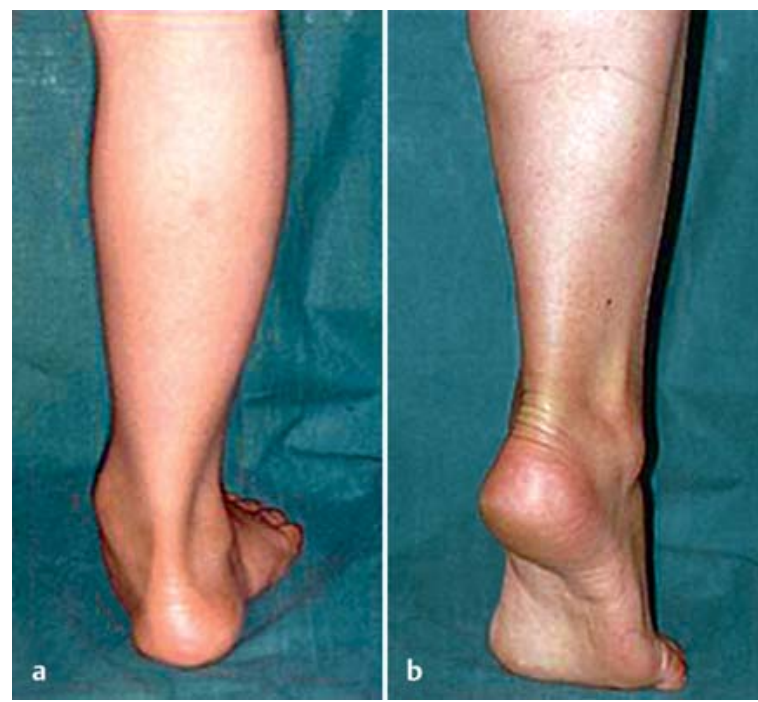

Abb. 10 - Single-Heel-Rise-Test. a Ausgangsstellung. $\mathbf{b}$ Bei flexiblem Knick-Senk-Fuß erfolgt die Aufrichtung des Längsgewölbes und die Geradestellung der Ferse aus dem Rückfußvalgus in Rektusposition.

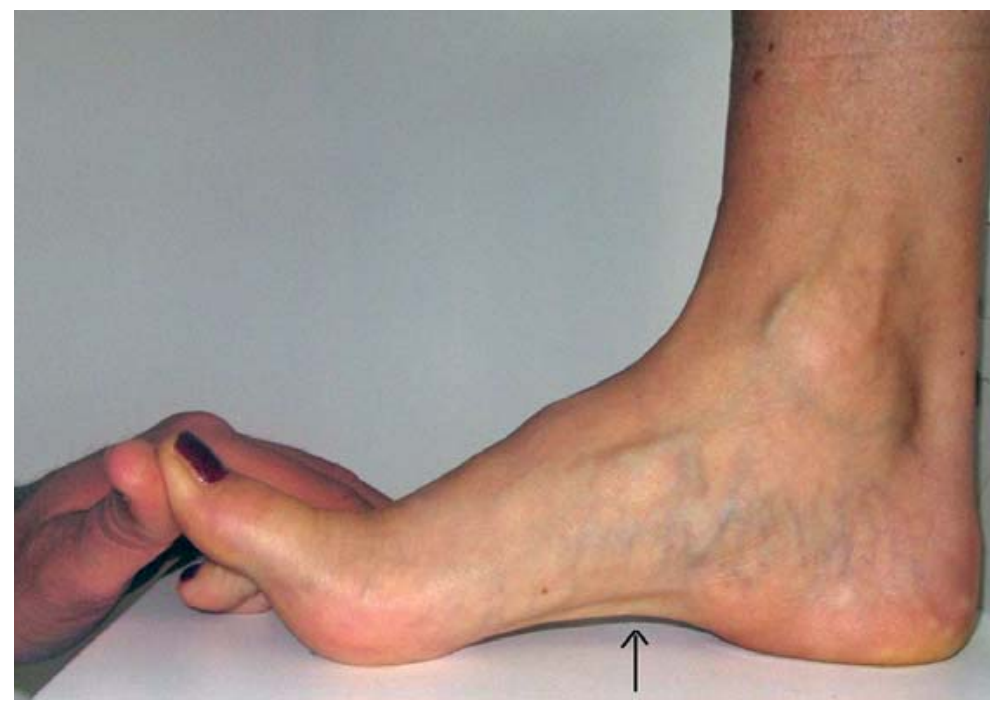

Abb. 11 - Windlass-Mechanismus: Test der ligamentären Verspannung der Fußsohle für die Aufrichtung des Längsgewölbes. Bei Dorsalextension der Zehe kommt es zur passiven Anspannung der Plantarfaszie und damit zum ligamentär vermittelten Windlass-Mechanismus. einer Tibialis-posterior-Insuffizienz III. und IV. Grades der Fall. Häufig sind ältere Patienten betroffen. Bei jüngeren Patienten sollte dem Verdacht einer Coalitio im Rückfuß nachgegangen werden. Am häufigsten finden sich talokalkaneare und talonavikulare Coalitiones.

Der Windlass-Mechanismus bewirkt, dass sich beim Heben des großen Zehs aufgrund der passiven Anspannung der Plantarfaszie und der Ligamente das Längsgewölbe ebenfalls aufrichtet (Abb. 11).
Der Hohlfuß hingegen zeigt ein überhöhtes Längsgewölbe und einen Rückfußvarus, oft eine angespannte Plantarfaszie und, je nachdem, ob ein Ballen- oder ein Hackenhohlfuß vorliegt, auch eine zusätzliche Spitzfußstellung. Auch die Kombination mit einem stark plantarisierten I. Strahl ist häufig. 


\section{Tabelle 4}

Klinische Stadieneinteilung der M.-tibialis-posterior-Dysfunktion [3,4].

\begin{tabular}{|c|c|c|c|c|}
\hline Insuffizienz & Johnson u. Strom & Johnson u. Strom & Johnson u. Strom & Myerson \\
\hline & Grad I & Grad II & Grad III & Grad IV \\
\hline M.-tibialis-posterior-Sehne & $\begin{array}{l}\text { Tenosynovitis oder } \\
\text { beginnende Degeneration } \\
\text { keine Elongation }\end{array}$ & $\begin{array}{l}\text { Elongation und Degeneration } \\
\text { oft chronische Teil- bis } \\
\text { Totalruptur }\end{array}$ & $\begin{array}{l}\text { Elongation und Degeneration } \\
\text { oft chronische Teil- bis } \\
\text { Totalruptur }\end{array}$ & $\begin{array}{l}\text { Elongation und Degeneration } \\
\text { oft chronische Totalruptur }\end{array}$ \\
\hline Knochendeformität & keine & $\begin{array}{l}\text { flexibel passiv reponierbare } \\
\text { Pes-planovalgus-Fehlstellung }\end{array}$ & $\begin{array}{l}\text { rigide nicht reponierbare } \\
\text { Pes-planovalgus-Fehlstellung }\end{array}$ & $\begin{array}{l}\text { rigide nicht reponierbare } \\
\text { Pes-planovalgus-Fehlstellung } \\
\text { Arthrose }\end{array}$ \\
\hline
\end{tabular}

Mit dem Coleman-Block-Test (Abb. 12) kann man durch Unterlagerung der Fußaußenkante feststellen, ob der Rückfußvarus ausgleichbar oder rigide ist und wie stark der Spitzfuß oder die Plantarisierung des I. Strahls ist.

Die Inhalte der Funktionsuntersuchung des Fußes sind in der Infobox „Zielgerichtete Diagnostik“ zusammengefasst.

Abb. 12 - Coleman-Block-Test. Durch eine laterale diagonal gelegene Außenranderhöhung kann die Varusstellung der Ferse beim flexiblen Hohlfuß korrigiert werden. Hierdurch kommt es zur Lateralisierung der Kraftlinie und dem orthograden Einleiten der Last auf den Rückfuß.

\begin{tabular}{|c|c|c|}
\hline \multicolumn{3}{|c|}{ Kraftgrad nach Janda [1]. } \\
\hline Kraftgrad & Prozent & $\begin{array}{l}\text { Kennzeichen: } \\
\text { aktive Bewegung gegen Widerstand }\end{array}$ \\
\hline $0 / 5$ & $0 \%$ & keine Aktivität \\
\hline $1 / 5$ & $10 \%$ & Muskelkontraktion ohne erkennbare Bewegung \\
\hline $2 / 5$ & $25 \%$ & Bewegung unter Ausschalten der Schwerkraft \\
\hline $3 / 5$ & $50 \%$ & Bewegen gegen die Schwerkraft \\
\hline $4 / 5$ & $75 \%$ & Bewegen gegen leichten äußeren Widerstand \\
\hline $5 / 5$ & $100 \%$ & Bewegen gegen starken äußeren Widerstand \\
\hline
\end{tabular}

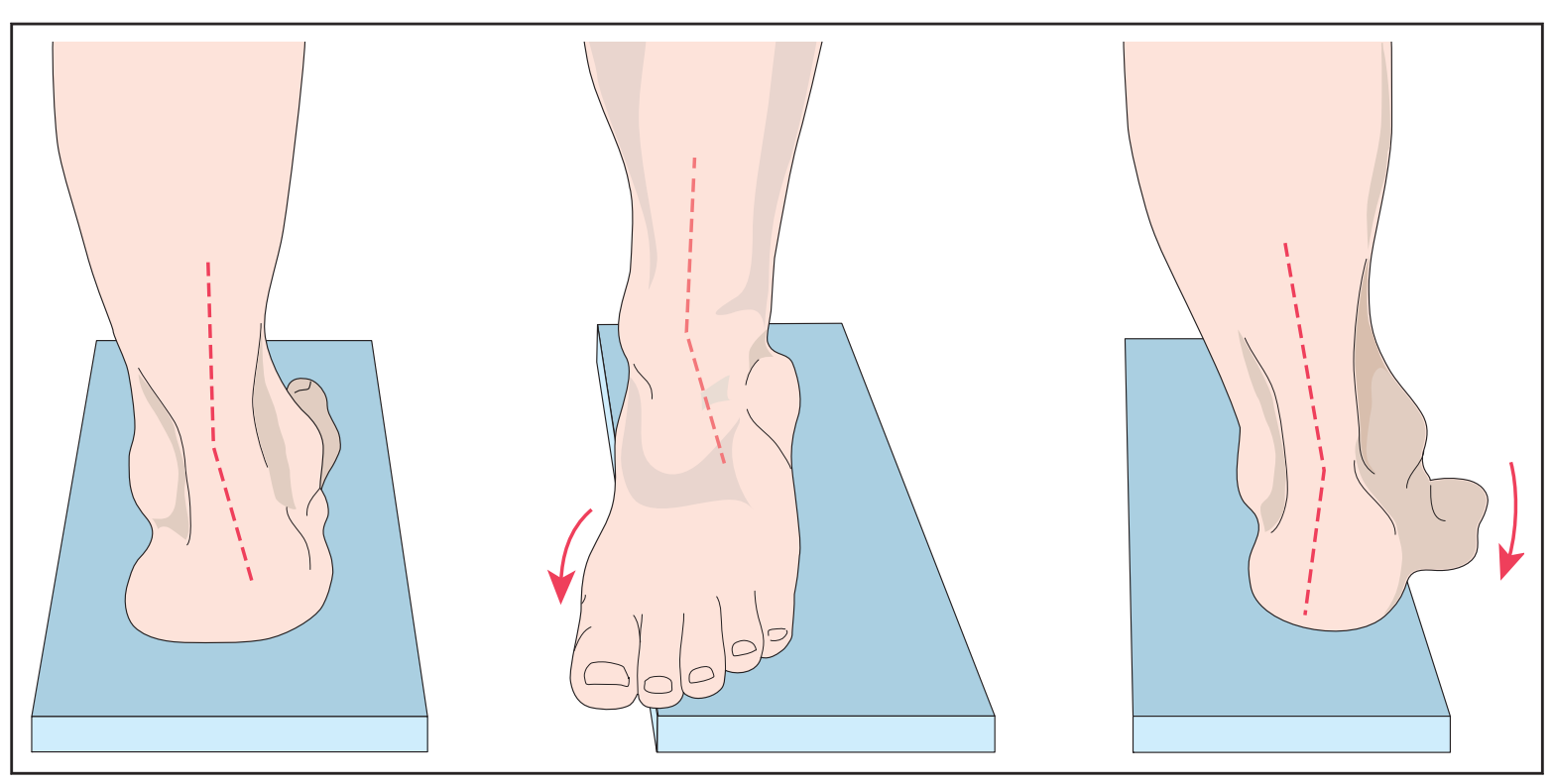




\section{Zielgerichtete Diagnostik}

\section{Inhalte der Funktionsuntersuchungen des Fußes}

- Stabilität/Hypermobilität TMT I

- Zehenspitzenstand beidseits und einseitig (Single-Heel-Rise-Test)

- Aufrichtung Rückfußachse

- Längsgewölbe

- Kraft in der Achillessehne

- S1

- Hackenstand

- Kraft in der M.-tibialis-anterior-Sehne

$-\mathrm{L} 5$

- Zehenspitzengang/Hackengang

- Ermüdung

- Seitendifferenz

- Beweglichkeit aktiv und passiv

- schmerzhaft/schmerzfrei

- Krepitation

- Kraftgrad OSG

- Heben/Senken

- großer Zeh: Heben/Senken

- Eversion (Pronation)/Inversion (Supination)

- Grind-Test

- Zehenstellung (flexibel, kontrakt)

- Grundgelenk (MTP)

- Mittelgelenk (PIP)

- Endgelenk (DIP)

- Push-up-Test

- Translationstest Grundgelenke Schublade

- Stabilität/Instabilität MTP-Gelenke

- Subluxation, Luxation

- Silfverskjöld-Test zum Feststellen einer Spitzfußstellung bei Verkürzung des M. gastrocnemius

- Windlass-Mechanismus bei Knick-Senk-Fuß zur Differenzierung einer flexiblen oder rigiden Fehlstellung

- Coleman-Block-Test zur Neutralisierung des Rückfußvarus bei flexiblem Hohlfuß

- Bandstabilität OSG

- laterale Aufklappbarkeit

- Talusvorschub

\section{Neurologischer Status}

Auch ohne in den Bereich der Neuroorthopädie oder Neurologie einzusteigen, ist es wichtig, Kenntnisse über eine grobe neurologische Untersuchung zu haben. Es ist nicht selten, dass Patienten mit einem primär unauffälligen Fuß die Sprechstunde aufsuchen, da sie stark unter den für sie unerklärlichen Symptomen einer Polyneuropathie leiden. Außer bei Diabetikern gibt es zahlreiche andere Gründe zur Entwicklung einer Polyneuropathie, allen voran das fortschreitende Lebensalter. Auch nach Chemotherapie, als Nebenwirkung von Medikamenten, aufgrund von Vitamin-B-Mangel, Nieren- und Schilddrüsenerkrankungen, Alkoholabusus, Infektionen, Autoimmunreaktionen etc. können neuropathische Symptome bzw. neuropathische Schmerzen auftreten.

- Leider gibt es - für den verzweifelten Patienten schlecht nachvollziehbar - viele idiopathische Fälle von Neuropathie.

Die Beschwerden sind typischerweise ein Brennen, Stechen und/oder Kribbeln an der Fußsohle oder den Zehen - auch nachts und ohne Belastung. Das Gefühl, auf Watte zu gehen, den Schuh als zu weit zu empfinden, und Gangunsicherheiten auf unebenen Boden gehören zu den Symptomen.

Davon abzugrenzen sind Beschwerden, die durch Irritation einer Nervenwurzel oder eines peripheren Nervs ausgelöst werden. Hier kann ebenfalls die Tiefen- oder Oberflächensensibilität gestört sein, gelegentlich auch die Motorik. Allerdings kann man in diesen Fällen die Zuordnung zu einem Dermatom bzw. dem Versorgungsgebiet des peripheren Nervs vornehmen. Zu der Anamnese gehört dann die Frage nach Rückenschmerzen, Operationen und Traumata auch oberhalb des OSG.

Mit den in der Infobox „Zielgerichtete Diagnostik“ zusammengefassten Untersuchungsmethoden kann man viele Informationen gewinnen, bevor man die Überweisung an den neurologischen Fachkollegen ausstellt. 


\begin{tabular}{l} 
Zielgerichtete Diagnostik \\
\hline Inhalte der neurologischen Untersuchung \\
des Fußes \\
\hline - Sensibilitätsverlust \\
- einem Dermatom zuzuordnen? \\
- strumpfförmig? \\
- Reflexe \\
- Achillessehne \\
- M. tibialis posterior \\
- Hoffmann-Tinel-Zeichen \\
- oberflächliche Nerven \\
- N. suralis \\
- Nn. peronaeus profundus und superficialis \\
- N. tibialis \\
• N. saphenus \\
- Vibration im Stimmgabeltest \\
- Rydel-Seiffer'sche Stimmgabel \\
- Semmes-Weinstein-Monofilament \\
- Sensibilitätsprüfung mit Druck von $01 \mathrm{~N}$ \\
- Temperaturempfinden \\
- Tipp Therm
\end{tabular}

\section{Podoskop}

Mit einem Podoskop kann man die Fußuntersuchung gut und ohne großen technischen Aufwand ergänzen. Die Fußbelastungszonen werden für den Untersucher und auch für den Patienten schnell und nachvollziehbar dargestellt. Fehlstellungen wie bei Knick-Senk-Fuß oder Hohlfuß können einfach über die Trittspur visualisiert werden (Abb. 13).

Hilfreich ist es zudem, den Patienten auf einfache Weise zeigen zu können, wann sie einen Bereich des Fußes nicht belasten, beispielsweise das Großzehengrundgelenk bei Arthrose. Die Patienten verlagern ihr Gewicht auf den Fußaußenrand und stützen sich mit dem Großzehenendglied ab. Daher kommt es, dass viele Patienten ihr arthrotisches Großzehengrundgelenk nicht schmerzhaft wahrnehmen und mit Schmerzen am V. Strahl in die Sprechstunde kommen.

Man kann auch eine dynamische Untersuchung durchführen, indem man den Patienten bittet, das Körpergewicht zu verlagern oder sich auf die Zehenspitzen zu stellen.
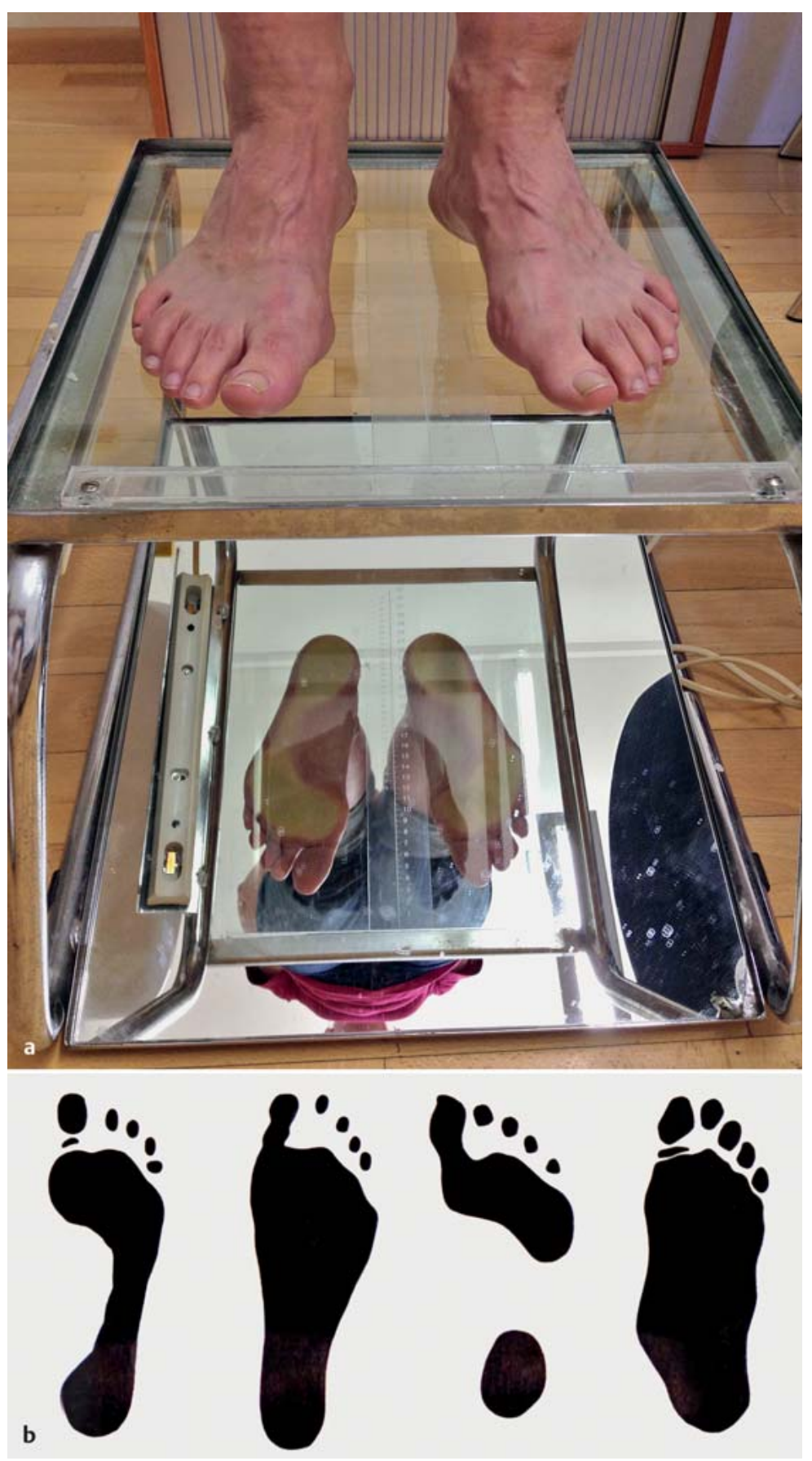

Abb. 13 - Podoskop. a Praktische Durchführung. b Trittspur von links nach rechts: normaler Fuß, Senkfuß („Plattfuß“), Hohlfuß, Knick-Senk-Fuß.

- Möchte man eine genauere Untersuchung der Druckverteilung statisch oder dynamisch durchführen, sollten ergänzend Fußdruckmessungen oder Ganganalysen durchgeführt werden. 


\section{Weiterführende apparative Diagnostik}

\section{[ Röntgen}

Im Anschluss an die Untersuchung des Fußes schließt sich je nach Befund meist die Röntgendiagnostik an. Die Standardanforderungen sind

- das Röntgen a.-p. mit ca. $15^{\circ}$-Röhrenkippung, um die Gelenke der Lisfranc-Linie darstellen zu können, und

- die seitliche Aufnahme.

Beide Aufnahmen werden im Stehen bei voll belastetem Fuß durchgeführt.

Ergänzende Aufnahmen wären

- die Schrägaufnahme,

- der Saltzmann-View zur Darstellung der Rückfußachse,

- die Sprinteraufnahme zur Darstellung der Sesambeine und der Ausrichtung der Metetarsaleköpfe oder

- seltenere Spezialaufnahmen.

\section{Zusätzliche bildgebende Verfahren}

Bedarfsweise sind zusätzlich CT, MRT, Szintigrafie, Sonografie oder andere Untersuchungen notwendig.

Interessenkonflikt: Die Autorin bestätigt, dass kein Interessenkonflikt vorliegt.

\section{Quellenangaben}

1 Viladot A. Der sog. Standardvorfuß. In: Wirth C], Hrsg. Vorfußdeformitäten. Berlin: Springer; 1993: 13

2 Niezold RM, Ferdini S. Klinische Untersuchung. In: Wirth C], Zichner L, Hrsg. Orthopädie und orthopädische Chirurgie. Band 8. Stuttgart: Thieme; 2002

3 Johnson KA, Storm DE. Tibialis posterior tendon dysfunction. Clin Orthop Relat Res 1989; 239: 196-206

4 Myerson MS. Adult acquired flatfoot deformity. Treatment of dysfunction of the posterior tibial tendon. J Bone Joint Surg Am 1996; 78: $780-792$

5 Janda V. Manuelle Muskelfunktionsdiagnostik. 4. Aufl. München: Urban \& Fischer; 2000

\section{Zum Weiterlesen und Vertiefen}

1 Fuhrmann RA, van Schoonhoven J. Degenerative Erkrankungen des 1. Zehenstrahls. Orthop Unfallchir up2date 2011; 6: 447-474

2 Sabo D. Vorfußchirurgie. Berlin: Springer; 2012

3 Sommer C. Fußchirurgie Ein praktischer Leitfaden. Wien: Springer; 2010

4 Sommer C. Operationstechniken in der Fußchirurgie. Wien: Springer; 2012

5 Wirth C], Zichner L, Hrsg. Fuß. In: Orthopädie und orthopädische Chirurgie, Band 8. Stuttgart: Thieme; 2002

6 Wülker N, Stephens MM, Cracchiolo A, Hrsg. Operationsatlas Fuß und Sprunggelenk. 2. Aufl. Stuttgart: Thieme; 2009

\section{Korrespondenzadresse}

Dr. med. Charlotte Hase

HELIOS Rosmann Klinik Breisach

Zeppelinstraße 37

79206 Breisach

Telefon: 07667/84-230

E-Mail: charlotte.hase@helios-kliniken.de 


\section{CME-Fragen}

\section{CME•thieme.de}

\section{CME-Teilnahme}

- Viel Erfolg bei Ihrer CME-Teilnahme unter http://cme.thieme.de

- Bitte informieren Sie sich vorab online über die Gültigkeitsdauer.

- Sollten Sie Fragen zur Online-Teilnahme haben, unter http://cme.thieme.de/hilfe finden Sie eine ausführliche Anleitung.

Einer der im Folgenden genannten Muskeln hat keinen Einfluss auf die Supination/Inversion des Fußes. Welcher?
A M. tibialis posterior
B M. tibialis anterior
C M. extensor hallucis longus
D M. flexor hallucis longus
E M. flexor digitorum longus

Wie viel Prozent Kraft entspricht Kraftgrad 2/5 nach Janda?

\section{A $10 \%$ \\ B $25 \%$ \\ C $50 \%$ \\ D $75 \%$ \\ E $100 \%$}

Welche(s) Gelenk(e) werden beim Push-up-Test geprüft?
A Hallux valgus

B Knick-Senk-Fuß

C Hohlfuß

D Pes adductus

E Hammerzehen
Eine der folgenden Aussagen zur Podoskopuntersuchung ist falsch. Welche?
A Die Durchführung einer dynamischen Untersuchung ist möglich.

B Die Fehlstellungen eines Knick-Senk-Fußes oder Hohlfußes können visualisiert werden.

C Die Podoskopuntersuchung ersetzt die Ganganalyse.

D Die Podoskopuntersuchung kann nachweisen, wenn das Großzehengrundgelenk bei Arthrose nicht belastet wird.

E Die Untersuchung ist ohne großen technischen Aufwand durchführbar.
A MTP-II-V-Gelenke

B PIP-Gelenke

C DIP-Gelenke

D MTP-I-Gelenk

E TMT-I-Gelenk 


\section{Grundlagen}

Der Silfverskjöld-Test ...

A ... misst die Kraft in der M.-tibialis-anterior-Sehne.

B ... weist die Stabilität/Instabilität der MTP-Gelenke nach.

C ... wird angewendet bei Knick-Senk-Fuß zur Differenzierung einer flexiblen versus rigiden Fehlstellung.

D ... dient zur Feststellung einer Spitzfußstellung bei Verkürzung des M. gastrocnemius.

E ... zeigt den Bewegungsumfang im OSG hinsichtlich Dorsalextension/ Plantarflexion an.

Worauf sollte bei der Untersuchung des Fußes in keinem Fall verzichtet werden?
A Grind-Test
B Vibrationstest mit der Stimmgabel
C Vergleich zur Gegenseite
D Push-up-Test
E Coleman-Block-Test

Welcher Nerv hat keine Äste am Fuß?
A N. tibialis
B N. peronaeus profundus
C N. peronaeus superficialis
D N. medianus
E N. suralis

Was ist keine typische Ursache einer Polyneuropathie?
A Diabetes mellitus
B Chemotherapie
C Vitamin-B ${ }_{12}$-Mangel
D Vitamin-E-Mangel
E fortschreitendes Lebensalter

Der Coleman-Block wird bei welcher Fußfehlstellung durchgeführt?
A Rückfuß valgus

B Hohlfuß

C Pes metatarsus adductus

D Schneiderballen

E Hallux limitus 